\title{
Sporadic Burkitt Lymphoma: A Case Report
}

\author{
Edgar Vargas Flores ${ }^{1 *}$, Claudia Leticia Avitia Raygoza ${ }^{2}$, Daniel Hernández Castañeda ${ }^{3}$, Jorge Arturo \\ Sánchez Garza ${ }^{3}$, Juan Carlos Quintana Cortéz ${ }^{1}$
}

${ }^{1}$ General Surgery Department, Hospital General de Zona 5, Instituto Mexicano del Seguro Social, Sonora, Mexico ${ }^{2}$ Hematology Department, Hospital General de Zona 5, Instituto Mexicano del Seguro Social, Sonora, Mexico ${ }^{3}$ Pathology Department, Hospital General de Zona 5, Instituto Mexicano del Seguro Social, Sonora, Mexico

*Corresponding Author: Edgar Vargas Flores, MD, General Surgery Department, Hospital General de Zona 5, Instituto Mexicano del Seguro Social, Sonora, Mexico. Tel: +52-5554995293, Email: eddgar868@gmail.com

Received September 3, 2018; Accepted October 21, 2018; Online Published January 28, 2019

\begin{abstract}
Introduction: Burkitt lymphoma is an aggressive subtype of Hodgkin lymphoma with a doubling time of 25 hours. It is characterized by a MYC gene coding alteration which results from a translocation of chromosome 8 on the MYC gene locus and immunoglobulin heavy-chain locus (IGH) on chromosome 14. Even though HIV infection is associated to an increased risk of Burkitt lymphoma, these type of malignancies are EBV-negative cases. Sporadic type Burkitt lymphoma is a rare clinical entity. Case Presentation: A 48-year-old female without any past medical history presented with a chief complaint of mild abdominal pain of 6 months duration located at the epigastrium which was not related to food ingestion. Nausea or vomiting were neither reported. She noted an acute increased abdominal pain over the last 4 hours before presentation, with irradiation to the right lower quadrant. She denied any Fever, night sweats and weight loss. She also reported hypermenorrhea in the last 6 months. During the physical examination, a palpable mass with $10 \mathrm{~cm}$ in diameter was found on right lower quadrant.

Conclusion: Sporadic Burkitt lymphoma is one of the rarest hematologic malignancies. The increased tumor size may be more than enough to show clinical or biochemical abnormalities such as serum lactate dehydrogenase (LDH) increased and tumor lysis.

Keywords: Lymphoma, Burkitt Lymphoma, Hematology
\end{abstract}

\section{Introduction}

Burkitt lymphoma is an aggressive subtype of Hodgkin lymphoma with a doubling time of 25 hours. It is characterized by a chromosome 8 translocation on the MYC gene locus and the heavy chain of the $\operatorname{IgH}$ immunoglobulin on chromosome 14. The sporadic form of Burkitt lymphoma is a rare clinical entity, present in only $30 \%$ of pediatric lymphomas and less than $1 \%$ of adult non-Hodgkin lymphomas in the United States. There is an increased incidence in younger individuals with a peak at 11 years and a peak of 30 years in adults. White patients also have an increased incidence, and males are usually more affected than women on a $4: 1$ ratio. ${ }^{1}$

There are 3 clinical forms of Burkitt lymphoma: endemic, sporadic, and immunodeficiency-related. There is an almost identical histological picture between these 3 forms. ${ }^{2,3}$

Sporadic Burkitt lymphoma commonly presents in adults younger than 35 years of age with mean age at time of diagnosis being 30 years. Caucasians are commonly more affected than African or Asian Americans., ${ }^{4,5}$

Patients diagnosed with Burkitt lymphoma occasionally manifest spontaneous tumor lysis syndrome with sudden elevations in lactate dehydrogenase and uric acid levels. Involvement of the terminal ileum and ascites may be present as well as that of the stomach, cecum and/or mesentery, kidneys, testicles, ovaries, breast, bone marrow, and/or central nervous system. Symptoms may vary and can also be associated with small bowel obstruction or lower gastrointestinal bleeding. They can also mimic appendicitis or intussusception. Up to $25 \%$ of patients present with involvement of the jaw or facial bones. Central nervous system or bone marrow involvement is present in $30 \%$ and $15 \%$ of cases, respectively, at the time of initial presentation, and this can also be a complication of recurrent or treatment-resistant disease. ${ }^{6}$

A histological diagnosis reveals medium-sized monomorphic cells with basophilic cytoplasm and an elevated proliferation of $\mathrm{Ki}-67+$ fraction that reaches almost $100 \%$ of its expression. ${ }^{3}$

Patients with Burkitt lymphoma require intensive and frequent multiple agent therapy with central nervous system prophylaxis. Rituximab is currently one of the drugs newly added to the treatment that has shown improved outcomes in patients.?

The report of the current case aimed to increase

Copyright $\odot 2019$ The Author(s). This is an open-access article distributed under the terms of the Creative Commons Attribution License (http:// creativecommons.org/licenses/by/4.0), which permits unrestricted use, distribution, and reproduction in any medium, provided the original work is properly cited. 
awareness among clinicians about one of the rarest forms of presentation of a somewhat common hematologic malignancy.

\section{Case Presentation}

A 48-year-old female with no past medical history presented with the chief complaint of mild abdominal pain with 6 months duration located at the epigastrium which was not related to food ingestion. No nausea or vomiting was reported. Fever, night sweats and weight loss were also denied. The patient stated she had an acute increase in abdominal pain intensity over the last 4 hours before presentation with irradiation to the right lower quadrant. She also reported hypermenorrhea. On physical examination, a firm, mobile, and painless palpable mass about $10 \mathrm{~cm}$ in diameter was found on her right lower quadrant. Lab studies showed Hb $9.3 \mathrm{mg} / \mathrm{dL} ; \mathrm{Hct} 29 \%$; $\mathrm{MCH}$ 29 pg; MCV 89 fl; leucocytes 7700/L, neutrophils 7003/L, lymphopenia of 560/L, monocytes $89 / \mathrm{L}$, eosinophils $38 / \mathrm{L}$, basophils 10/L, and lactate dehydrogenase $1162 \mathrm{UI} / \mathrm{L}$. HIV and HBV tests were both negative.

A CT scan of the abdomen showed multiple retroperitoneal lymph node conglomerates, right hydronephrosis, and bilateral ovarian enlargement as the most relevant findings (Figure 1). The patient was sent to the operating room for a laparotomy where abundant ascites fluid was obtained and multiple visceral and enlarged retroperitoneal lymph nodes were palpated. A right oophorectomy was performed and samples were sent to pathology. The patient underwent an uneventful recovery for the first 24 hours; however, on post-operative day 2, she reported shortness of breath. A chest X ray showed the presence of bilateral pleural effusions which were initially managed with a loop diuretic. Finally, the pathology analysis revealed a diagnosis of a CD20+, CD10+, bcl-6 -, CD3 -, TDT Ki67 98\% Burkitt lymphoma (Figure 2). A chemotherapy approach was begun with CODOX-M/IVAC. After the second chemotherapy cycle, a surveillance CT scan was performed and showed the absence of neoplastic activity. Long-term surveillance (2 years) showed no recurrent disease.

\section{Discussion}

Sporadic Burkitt lymphoma is an infrequent hematologic disorder accounting for just $1 \%$ of non-Hodgkin lymphomas in adults. ${ }^{1,8}$ The presence of gynecologic symptoms as well as the absence of tumor lysis syndrome make the case presented herein an exceptional presentation for non-Hodgkin lymphoma. ${ }^{9}$ The rapid and constant cellular growth of the intra-abdominal tumors was clinically and biochemically evidenced by the surge of lactate dehydrogenase. ${ }^{6}$

A laparotomy was performed on this patient mainly for biopsy purposes and not as a therapeutic resolution. ${ }^{10}$ Even though some studies suggest that Burkitt lymphoma cannot originate on the ovary because of a lack of lymphoid tissue, it has been found that up to $54 \%$ of patients present small

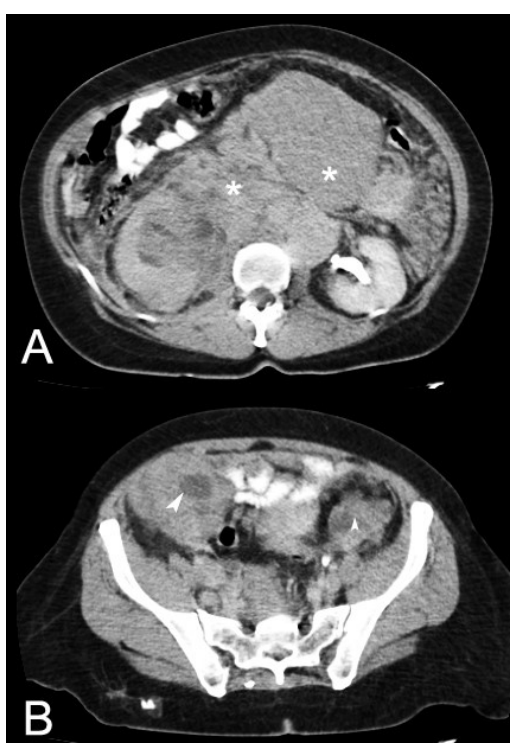

Figure 1. A. Contrast-enhanced CT Scan of the Abdomen Showing Retroperitoneal Lymph Node Enlargement (asterisks); B. Bilateral Ovarian Enlargement (arrows).

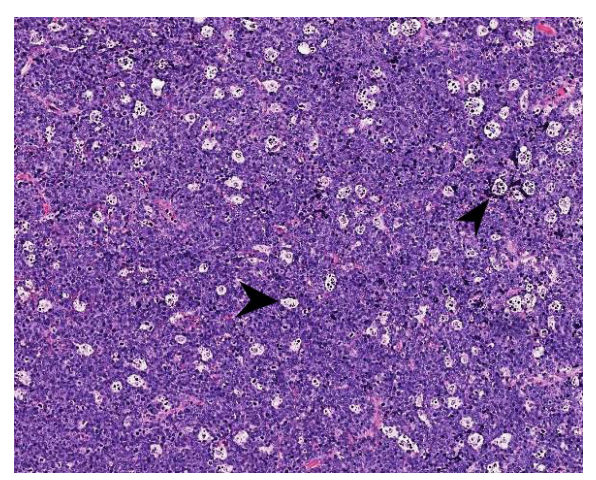

Figure 2. Diffuse Architecture, Medium-Sized Lymphocytes With Scant Cytoplasm, and Presence of Numerous Tangible-Body Macrophages Representing Histiocytes Phagocytizing Apoptotic Cell Debris (black arrows).

groups of lymphocytes on the blood vessels that surround the ovary and the luteal body. Non-Hodgkin lymphoma rarely arises on gynecologic anatomy, but when it does (as in the present case), the ovary is the most common anatomic site and causes female hormone disturbances. Rare cases of sporadic Burkitt lymphoma have been found in specific anatomic regions of the head such as those reported in Europe and Asia by Aslan and Patankar et al, respectively. ${ }^{11,12}$ In some low-income health centers where minimally invasive surgical approaches or imaging guided biopsies are not always available, a rapid onset of action with more aggressive measures may clearly help a patient when an uncertain diagnosis arises, increasing the chances of survival and diminishing the risk for potential complications in late-onset treatment.

\section{Conclusion}

Increased tumor size may be more than enough to show clinical or biochemical abnormalities such as tumor lysis 
and elevated serum lactate dehydrogenase. Nevertheless, the presented case shows that these abnormalities may not always be the rule. Prompt clinical suspicion as well as early diagnosis and initiation of treatment allows the clinician a quick start on chemotherapy, decreasing the chance of complications and morbidity such as tumor lysis syndrome and increasing the survival rate.

\section{Authors' Contributions}

All authors contributed equally to this study.

\section{Conflict of Interest Disclosures}

The authors declare that they have no conflicts of interest.

\section{Ethical Approval}

For ethical approval of this case report, informed consent was obtained from the patient.

\section{References}

1. Jacobson C, LaCasce A. How I treat Burkitt lymphoma in adults. Blood. 2014;124(19):2913-2920. doi:10.1182/ blood-2014-06-538504.

2. Swerdlow SH, Campo E, Harris NL, et al. WHO Classification of Tumours of Haematopoietic and Lymphoid Tissues. Lyon: IARC Press; 2008.

3. Swerdlow SH, Campo E, Pileri SA, et al. The 2016 revision of the World Health Organization classification of lymphoid neoplasms. Blood. 2016;127(20):2375-2390. doi:10.1182/ blood-2016-01-643569.

4. Morton LM, Wang SS, Devesa SS, Hartge P, Weisenburger DD,
Linet MS. Lymphoma incidence patterns by WHO subtype in the United States, 1992-2001. Blood. 2006;107(1):265-276. doi:10.1182/blood-2005-06-2508.

5. Armitage JO, Weisenburger DD. New approach to classifying non-Hodgkin's lymphomas: clinical features of the major histologic subtypes. Non-Hodgkin's Lymphoma Classification Project. J Clin Oncol. 1998;16(8):2780-2795. doi:10.1200/ JCO.1998.16.8.2780.

6. Robbiani DF, Deroubaix S, Feldhahn N, et al. Plasmodium Infection Promotes Genomic Instability and AID-Dependent B Cell Lymphoma. Cell. 2015;162(4):727-737. doi:10.1016/j. cell.2015.07.019.

7. Bishop PC, Rao VK, Wilson WH. Burkitt's lymphoma: molecular pathogenesis and treatment. Cancer Invest. 2000;18(6):574583. doi:10.3109/07357900009012197.

8. Smith A, Howell D, Patmore R, Jack A, Roman E. Incidence of haematological malignancy by sub-type: a report from the Haematological Malignancy Research Network. Br J Cancer. 2011;105(11):1684-1692. doi:10.1038/bjc.2011.450.

9. Lenz G, Staudt LM. Aggressive lymphomas. N Engl J Med. 2010;362(15):1417-1429. doi:10.1056/NEJMra0807082.

10. Stepniak A, Czuczwar P, Szkodziak P, Wozniakowska E, Wozniak S, Paszkowski T. Primary ovarian Burkitt's lymphoma: a rare oncological problem in gynaecology: a review of literature. Arch Gynecol Obstet. 2017;296(4):653-660. doi:10.1007/s00404-017-4478-6.

11. Aslan G. Unusual presentation of sporadic Burkitt's lymphoma originating from the nasal septum: a case report. J Med Case Rep. 2013;7:60. doi:10.1186/1752-1947-7-60.

12. Patankar S, Venkatraman P, Sridharan G, Kane S. Burkitt's lymphoma of maxillary gingiva: A case report. World J Clin Cases. 2015;3(12):1011-1016. doi:10.12998/wjcc. v3.i12.1011 